\title{
TEE 2005: an advanced software tool for the modelling of direct impacts of transport system
}

\author{
B. B. Bellés ${ }^{1}$, A. Agostini ${ }^{2}$, S. Carrese ${ }^{1}$, M. Lelli ${ }^{2}$, E. Negrenti ${ }^{2}$ \\ \& A. Parenti ${ }^{3}$ \\ ${ }^{1}$ University of Roma TRE, Italy \\ ${ }^{2}$ ENEA ENE TEC CR Casaccia, Italy \\ ${ }^{3}$ ASTRAN srl, Italy
}

\begin{abstract}
The new version of the TEE (Transport Energy Environment) model has been developed in the frame of the FP5 ISHTAR and HEARTS Projects with several features for better analysing transport related direct impacts, taking into account vehicles kinematics, cold start emissions distribution, parking processes, noise emissions and accident occurrence. Specific efforts were dedicated to the modelling of the effects of vehicles kinematics on hot emissions, where the software calculates link emissions by adopting average speed based functions, instantaneous emissions, or the innovative 'kinematics correction functions' model, and the modelling of parking processes which are relevant for cold start and evaporative emissions.
\end{abstract}

Keywords: traffic emission, impacts of transport system, parking modelling, noise emissions, accident.

\section{Introduction}

Emissions from transport vehicles are modelled in a number of different ways, but this variability includes some 'fundamental rules' that give the 'essence' of emissions calculation: emissions are in practice the sum of at least two main components: 'hot emissions' and 'cold start emissions'. If the modeller is interested in VOC emissions, then also the 'evaporative term' has to be added. Hot emissions are the emissions emitted when engine and abatement devices have reached a regime temperature. They are influenced by a number of parameters: vehicle kinematics, gradient of the road, altitude, maintenance level, 
vehicle age, vehicle loading, and electric loads. Normally models refer to hot emissions as a function of kinematics (e.g. average speed or instantaneous speed and acceleration) and then multiply the 'ideal' hot emission value for a number of corrective factors taking into account the other mentioned parameters. Cold Start emissions are the emissions emitted from the start up until the vehicle reaches an almost steady state thermal condition. The cold start emissions affect in practice the first 3 or $4 \mathrm{kms}$ of trip and are particularly relevant for catalyst vehicles: for CO and VOC the 'cold start emission' is roughly 10 times higher than the hot emission term.

Evaporative emissions are the emissions of unburned fuel from the 'weak points' of the vehicle: tank and canister. Current classic modelling recognises three different contributions to evaporative emissions: a) 'running emissions', emitted when vehicles are driven (emissions at tank level), b) 'Hot Soak' emissions, emitted from the canister at trip conclusion, and 'Diurnal emissions', emitted at tank level by vehicles already parked. Evaporative emissions are a relevant fraction of total transport related VOC emissions, and so have a major role in the planning of measures for reducing VOC related pollution (e.g. critical benzene air pollution in Southern European cities). Two fundamental paths for increasing the accuracy of link based emission models are the more realistic modelling of vehicle kinematics and parking processes. Until now, very limited knowledge exists regarding the various aspects of vehicle parking and its environmental effects.

\section{Methodology}

The new version of TEE model allows to better analyse transport related direct impacts, taking into account vehicle kinematics, cold emission distribution, parking processes, noise emissions and accident occurrence. As it regards vehicles kinematics, TEE calculates link emissions by adopting different options: average speed based emissions, instantaneous emissions, and the innovative 'kinematics correction functions' model describing both traffic flow condition and vehicle behaviour. The adoption of the kinematics correction has given very good results in the prediction of CO concentration levels in the city of Rome. For cold start emissions distribution, TEE now offers alternative solutions based on defaults of cold percentage depending on area type and day hour, or on the user input of either a link based cold fraction or the link related driven distance from trip origin. The parking sub-model provides a meaningful treatment of traffic flows from and to parking areas and so allows to better locate cold start vehicles emissions and evaporative emissions in space and time. The software now includes two fully new models: the noise emission model and the accident occurrence model. The former is sensible to vehicle speed and heavy duty vehicles presence. The accident model calculates the number of accidents involving only vehicles or vehicles and pedestrians and splits them by severity. These new models broaden the scope of TEE software from pure 'consumption and emissions' modelling to the area of the direct impacts of transport systems. Furthermore a new routine for calculating the effect of the electric loads has been 
inserted. These developments have been realised in the frame of the FP5 ISHTAR Project.

One of the most relevant developments for the accurate estimate of pollutant emissions distribution at urban level is the realisation of an advanced parking model. Parking policy plays an important role in the traffic system and it affects the level of traffic congestion and environmental quality in most cities. Parking search and insertion are a perceptible source of congestion and pollution: half of cars driving downtown during peak periods in heavy congested areas are cruising for parking [2] and searching time is usually a high rate of total travel time [1]. However there is a lack of existing studies and data in order to evaluate the influence of parking in pollutant emissions. So, special attention must be given to vehicle parking and its environmental effects in the scientific research community as well as urban planners and consulting engineers.

This work focuses on a specific parking model for the accurate determination of emissions due to parking inlet and outlet processes as represented within the TEE modelling framework. Additional emissions from parking procedures can be quantified by traffic simulation models with additional computer models for emission calculations as TEE. The TEE model link representation is characterized by three 'flow modes' corresponding to transit, parking and inserting vehicles. The behaviour of the parking and inserting flows is described by means of simplified speed cycles, based on the kinematics of the vehicle during the parking and the insertion phases. The main parameters defining the shape of these cycles are searching speed, searching time and time of warm up. Parking search phase is represented by dedicated 'searching speed' and 'searching time' models. The searching speed model and the searching time model are further divided into two sub-models depending on the category of parking: on-street and off-street. In the on-street parking case searching speed is modelled by a fuzzy model as a function of the mean linear vehicle density and the occupation rate of the parking facility. Searching time for kerbside parking along the street uses a probabilistic approach to calculate the searching time based on the occupation rate of the link connected parking areas. In the off street case a detailed simulation of choice of parking place is proposed to reproduce the vehicle movements from entrance to parking place. From the results of this simulation we are able to determine speed and searching time. As it regards the inserting phase, a large amount of pollutants is emitted due to the cold start effect. A set of models have been proposed in order to calculate the time employed by a car to be fully warmed up. Applications of the models showed that the parking model approach improves the sensibility of the TEE software. These specific models are being integrated in the TEE 2005 Model of ENEA in the framework of the EC FP5 HEARTS Project and will be applied in a European City in 2005.

\section{Results and discussion}

The two main developments recently obtained are here discussed: the kinematics modelling for transit flows and the modelling of parking. 
One of the promising alternatives to the classically 'macroscopic' average speed emissions approach aims at obtaining a reasonably adequate kinematics description on the basis of easily available input data: the link average speed itself, a congestion indicator such as the 'lane flow density', link length and the fraction of green time at the intersection at the end of the link. The traffic density is used for calculating the fractions of time spent during cruising, acceleration, deceleration and idling phases. For low densities, traffic is smooth, very limited interactions occur among vehicles and all the time is spent in the cruise phase. For higher density values accelerations induced by interactions start, thus reducing time available for cruising. When density exceeds a threshold value, spontaneous breakdown occurs and short idling events take place. As density finally approaches the upper limit value we observe a fast decline of cruise time, a progressive reduction of time spent in accelerations and the rise of idling time until traffic is blocked at the density saturation value. The time spent during acceleration and deceleration is used to estimate the number of acceleration episodes on the basis of a driver behaviour hypothesis and acceleration rates derived from measured data. The minimum speed along the speed cycle can also be calculated from these data. The knowledge of the average speed and the time spent in cruising and in the other driving phases allows also the cycle cruising speed to be calculated. The overall result is a 'reconstructed' speed profile characterised by: a time interval spent in idling (only for high densities or presence of a traffic light), some accelerations and deceleration episodes between the minimum speed and the cruise speed, and a time interval spent at 'cruise' speed. This relatively simple solution describes the likely speed cycle far from the traffic light that is supposed (if any) at link end. The 'Kinematics Correction Function' (KCF) Model makes extensive use of the 'reconstructed cycle' algorithm. This approach assumes that the effect of speed variability can be expressed by means of a 'kinematics correction function'. The 'corrected' emission ' $E$ ' is obtained as the product of the 'average speed emission ' $\mathrm{e}$ ' and the 'kinematics correction factor' $\mathrm{KCF}$ :

$$
E=e(v) \times K C F
$$

The KCF can be derived from a set of emission calculations in which both the 'reconstructed cycle' model and the average speed correlation are used. In order to derive a function of general applicability, the authors had to define a matrix of possible traffic conditions. By considering a discrete number of average speeds, of 'green time percent', of congestion levels represented by traffic density, and of different link lengths, it was possible to define a four-dimensional matrix of theoretical traffic situations for which the fuel consumption and the emissions of $\mathrm{CO}$ and VOC were calculated with both the approaches. This way the influence of the speed cycle can be quantified and the characterisation of the KCF as a function of speed, density, green time fraction and link length can be performed. The matrix used in the most recent recalculation of the KCF includes over 1300 different traffic conditions. The correlation between the $\mathrm{KCF}$ and the four input parameters was searched as the product of four independent functions:

$$
K C F=K O \times d(D) \times g(G) \times 1(L) \times v(V)
$$


where $\mathrm{d}, \mathrm{g}, \mathrm{l}$, and $\mathrm{v}$ are classic mathematical functions of the variables $\mathrm{D}$ (traffic lane density, in vehicles/Km), G (green time fraction), L (length, in $\mathrm{Km}$ ) and $\mathrm{V}$ (Average Link Speed in $\mathrm{Km} / \mathrm{hr}$ ), and $\mathrm{K} 0$ represents the value of the $\mathrm{KCF}$ coefficient for 'central' values of the four input variables.

TEE model adopting this correction function was assessed against $\mathrm{CO}$ measurements in Rome in the frame of the FP5 HEAVEN and ISHTAR Projects. Estimated CO levels were compared with the measured values in the Viale Libia monitoring station. Significant differences were found between the use of the KCF correction and the simple use of the COPERT III functions for hot emissions. The use of the correction largely provided the best results (see Fig 1). The daily profiles are predicted with very good approximation. The correlation of data calculated and measured is remarkably high (0.85). The calculated CO level without the adoption of the KCF correlation would have been sensibly lower (for the case of $\mathrm{CO}$ the KCF function ranges normally between 1 and 3 ).

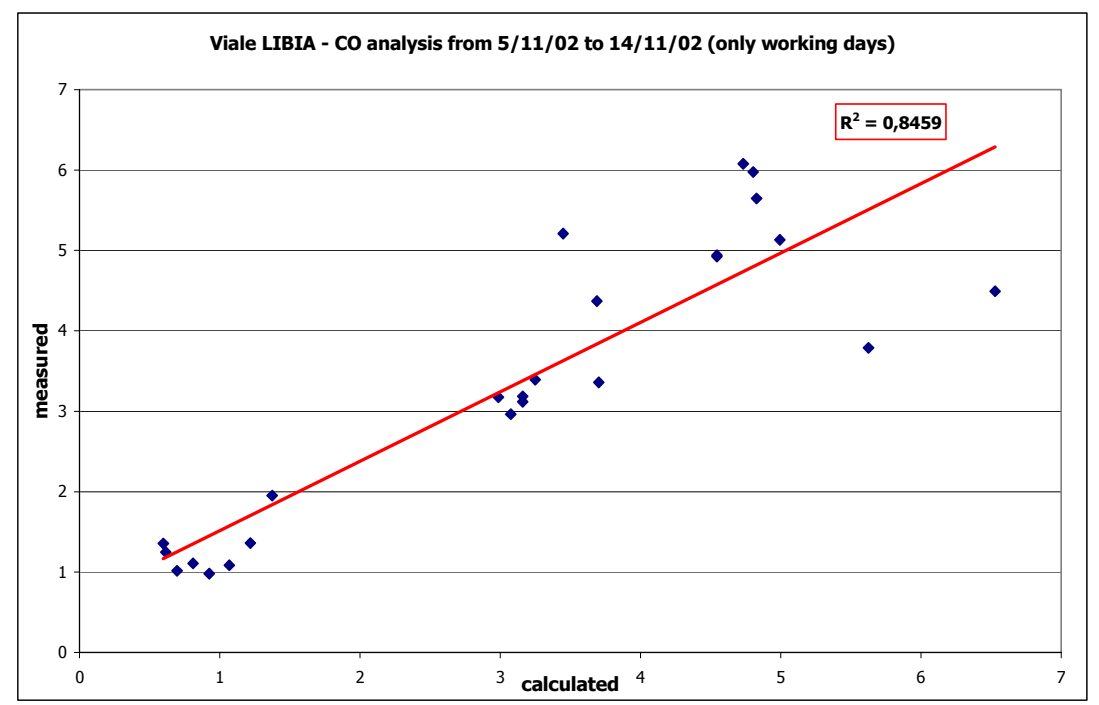

Figure 1: Correlation of measured and calculated CO concentrations $(\mathrm{mg} / \mathrm{m} 3)$ at Viale Libia (working days data between 5 and 14 November 2002 - Courtesy of STA SpA Rome - 2002).

Parking models have been created to simulate parking inlet and outlet processes and quantify the parking impacts on air pollution. Vehicle energy consumption and emissions related to parking need to be assessed and quantified. Parking search phase is represented by searching speed model and searching time model for on street and off street parking. Searching speed model for on street cases allows one to evaluate the speed adopted by the user in the parking search and it is related to meaningful variables of link and parking state provided as input by a traffic model (occupation rate and linear vehicle density). The model is based on fuzzy logic. The development of a fuzzy logic is well 
known and it has been thoroughly described elsewhere [10]. The assumptions are that driver's behaviour can be described by a set of rules IF-THEN, and the variables link flow density and parking occupation rate are described by a number of membership functions that attribute at each value a real number $\in[0,1]$ describing the degree at which real number belongs to fuzzy set. The output variable is searching speed that increases with link flow speed and decreases for higher values of the occupation rate for the same values of link speed. The output allows TEE to built the simplified cycle (speed against time) and calculate the additional emissions.

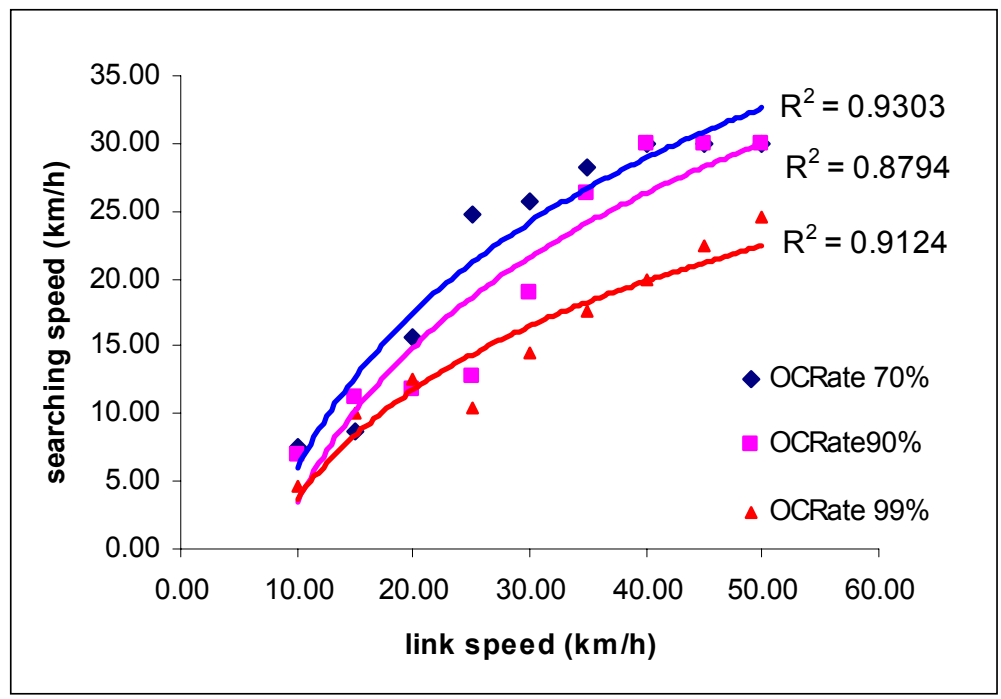

Figure 2: Relationship between searching speed as function of flow speed for several OC Rates.

Searching time model evaluates the time employed by a driver from the moment he decides to park up to he finds an available space to do it. Parking models and approaches present in literature $[3,4,5]$ has been used to investigate parking policies in a network framework. This research is meanwhile addressed to study the searching time in a link and, therefore it cannot be represented with network models. Searching time is calculated assuming a uniform distribution of the parking along a link. Once the user reaches this link, he cruises the link at searching speed and examines sequentially all the places up until he finds a free one that satisfies all the conditions. The behavioural model of the searching time is then based on the analysis of probability of attempting that a driver must carry out before finding a useful place to park. The relationship of searching time is the following

$$
T_{\text {searching }}=\mu \frac{L_{p}}{V_{\text {searching }}}=\frac{L_{p}}{V_{\text {searching }}} \cdot \frac{N+1}{o c c_{2}-o c c_{1}} \cdot \log \left(\frac{N+1-o c c_{2}}{N+1-o c c_{1}}\right)
$$


where $\mu$ is the mean value of attempting, $\mathrm{N}$ is the total parking places, Lp is the length of considered parking place, Occl is the number of occupied places in the beginning of the study interval and Occ 2 at the end of interval.

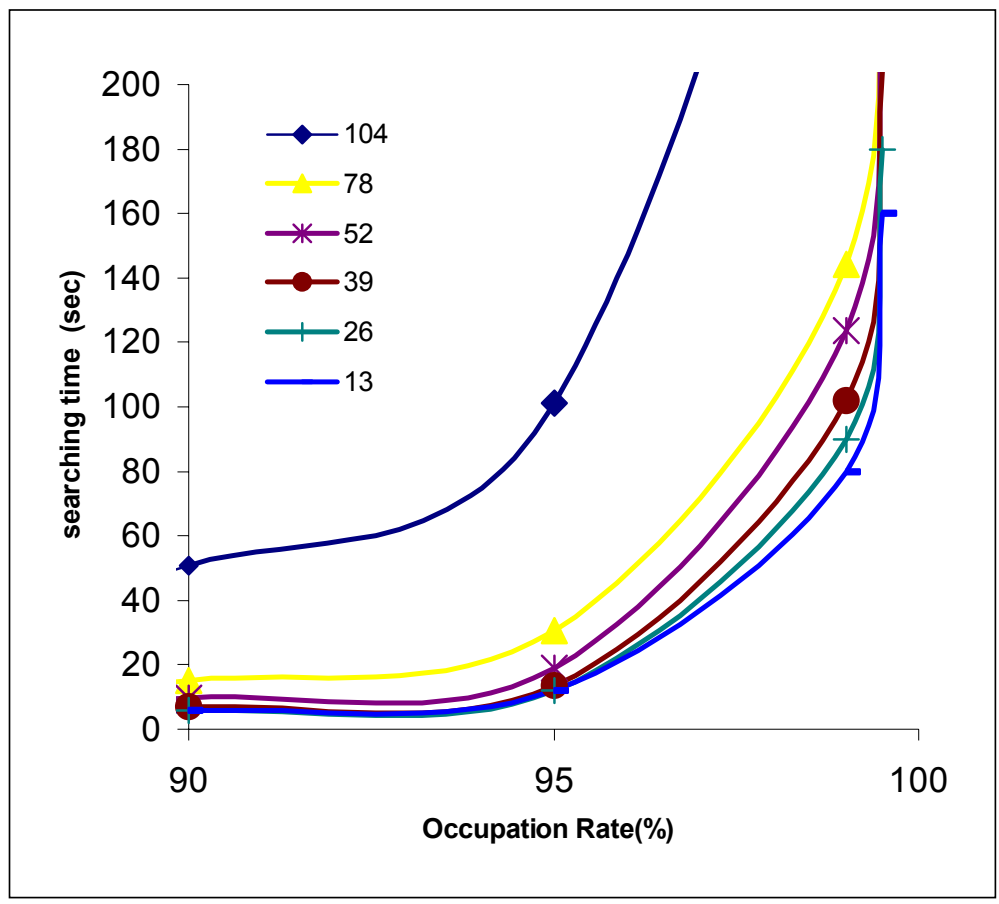

Figure 3: $\quad$ Searching time and occupation rate for several densities.

In the off street cases, searching speed depends on occupancy rate and geometry characteristics describing parking lanes. Young [9] has shown that driver in parking establishment have a desired speed that is influenced by the characteristics of road, particularly its length. For this purpose data supplied by Young paper has been fundamental in the modelling of vehicle flow speed on parking facilities. Relationship based on length of road and occupancy rate has been found and used to calculate the searching speed. On the other hand, searching time in a parking establishment has been studied analysing the parking lot choice. The choice of parking place consists in two level decisions: the choice of isle inside the parking and the choice of the space inside the isle. The choice of isle is modelled with a Logit model calibrated by Van der Waerden et al. [8] and it supplies the distribution of parking demand among the isles. Variables used in the Logit model are the distance from each isle to the ticket machine, exits to shopping and working areas, park \& ride installations and the entrance of parking. The first decision level requires also the knowledge of the link-path 
incidence matrix. Once the user has chosen the isle maximizing its utility, the time employed inside the isle is calculated with the same probabilistic model used in the onstreet case. So, the searching time is the sum of time required to reach the isle and the time employed searching a free space inside of it.

As regards the inserting phase a suite of numerical models have been developed in order to predict time of warm up, catalyst time of light off and efficiency of catalyst conversion from the knowledge of the motor vehicle dynamics, fuel consumption, temperature of air and catalyst geometry. The model supplies a detailed transient simulation of the engine warm up period from initial ambient conditions to a fully warmed up state and the main output variables are the working temperature of engine and the temperature of the catalyst converter second by second. The integration of these models into TEE Code allows the additional emissions during the cold start and the percentage of cold vehicles to be calculated.

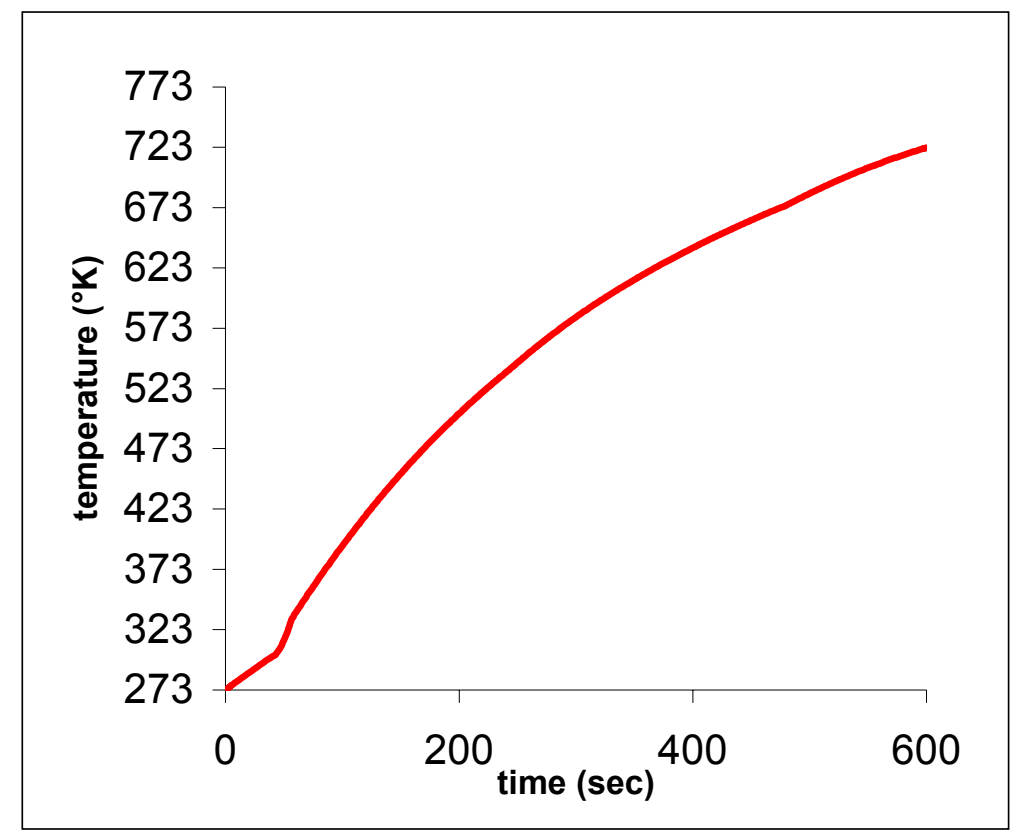

Figure 4: Catalyst temperature with ambient temperature of $20^{\circ} \mathrm{C}$.

\section{Conclusions}

New approaches have been developed for describing the effects of vehicle kinematics both in the transit flows and in the parking processes. These new models reduce the uncertainties in the description of the spatial and temporal distribution of emissions in all its main components: hot, cold start and evaporative. 


\section{Acknowledgements}

Acknowledgements are here made to the colleagues at ASTRAN s.r.l for the support in the development the TEE software and to the ISHTAR partners and sub-contractors (APSIS Athens, Graz Technical University, DITS at Rome University 1) for their assessment work.

\section{References}

[1] Auxhausen and Polak (1990). Choice of parking: Stated preference approach. Paper no. 560 of the TSU. Oxford University.

[2] Anderson and Palma (2004). The Economics of on-street parking: Road Congestion and Driver Search. Journal of Urban Economics, 55(2004) $1-20$.

[3] Bifulco C. (1993). A stochastic user equilibrium assignment model for the evaluation of parking models. European Journal of operation research (71) 269-287.

[4] Carrese S, Gori S. (1996). Relationship between parking location and traffic flows in urban areas. Advanced methods in Transportation Analysis.

[5] Coppola, P. (2001). A joint model of mode/parking choice with elastic parking demand. Transportation Planning 85-104.

[6] Hoglung, Paul (2004). Parking, energy consumption and air pollution. Science of the Total Environment 334-335 (2004) 39-45.

[7] Negrenti, E. (1996) TEE: ENEA traffic emissions and energetic model micro-scale applications. The science of the total environment 189/190167-174.

[8] Van der Waerden, P et al (2003) Travelers Micro-behaviors at Parking Lots: A model of Parking Choice Behaviors. TRB 2003 Annual Meeting CD-ROM.

[9] Young, W. (1988). The distribution of speed in parking facilities. Traffic Engineering \& Control. October 1988 - Vol. 29, No10. 504-513.

[10] Zadeh, L.A. (1978). Fuzzy Sets a basis for a theory of probability. Fuzzy sets and systems. 1,3-28. 